\title{
Recombinant activated factor seven in pediatric cardiac surgery- does thrombotic risk outweigh hemostatic benefit?
}

\author{
John P. Scott \\ Department of Anesthesiology and Pediatrics, Sections of Pediatric Anesthesiology and Pediatric Critical Care, Medical College of Wisconsin, \\ Milwaukee, WI, USA \\ Correspondence to: John P. Scott. Department of Anesthesiology and Pediatrics, Sections of Pediatric Anesthesiology and Pediatric Critical Care, \\ Medical College of Wisconsin, 9000 W. Wisconsin Ave. PO Box 1997, Milwaukee, WI 53201-1997, USA. Email: scottjake@mcw.edu. \\ Provenance: This is an invited article commissioned by the Section Editor, Xicheng Deng (Department of Cardiothoracic Surgery, Hunan Children's \\ Hospital, Changsha, China). \\ Comment on: Christoff AS, Winlaw DS, Curtin J, et al. Recombinant activated factor VII in neonatal cardiac surgery. Eur J Cardiothorac Surg \\ 2019;55:817-22.
}

Submitted Sep 17, 2019. Accepted for publication Oct 24, 2019.

doi: $10.21037 /$ tp.2019.10.04

View this article at: http://dx.doi.org/10.21037/tp.2019.10.04

Therapies employed to attenuate perioperative bleeding following cardiopulmonary bypass (CPB) in children include antifibrinolytic therapy, allogenic blood product transfusion, and recombinant factor concentrates. Recombinant activated factor seven (rFVIIa) (Novoseven RT, Novo Nordisk A/S, Denmark) is a factor concentrate with federal drug administration (FDA) approval for treatment of bleeding in Hemophilia A and B with inhibitors, Congenital Factor Seven deficiency, and Glanzmann's Thrombasthenia. Off label rFVIIa is frequently administered as rescue therapy for recalcitrant perioperative bleeding post-CPB and has been linked to decreased transfusion requirements, chest tube output, and need for surgical re-exploration (1-3). However, concerns remain regarding the safety of rFVIIa in this setting, specifically related the development of thrombotic complications (4).

In a recent issue of The European Journal of CardioThoracic Surgery, Christoff and colleagues report the results of a single-center retrospective study of perioperative rFVIIa use following neonatal congenital cardiac surgery (5). Their aim was to assess whether rFVIIa treatment was associated with increased clinical thrombosis defined as any event presenting with physical exam findings. Neonates treated with rFVIIa were compared with those who were not. Overall $31 \%$ of neonates received rFVIIa, including notably high rates following arterial switch procedures $(27 \%)$ and Norwood procedures $(57 \%)$. The overall incidence of clinical thrombosis was $11 \%$, and $22 \%$ of neonates treated with rFVIIa developed thrombosis compared to $6 \%$ of non-treated neonates. A significant association was observed between rFVIIa treatment and clinical thrombosis $\mathrm{OR}=4.4$ (95\% CI, 2.3-8.4; $\mathrm{P}<0.001)$. Almost all thromboses (96\%) were detected in vessels with indwelling vascular lines. After adjusting for severity of illness, rFVIIa was associated with significantly more thrombosis. Neonates undergoing Norwood procedures who were treated with rFVIIa had increased risk of thrombosis (odds ratio 2.3; 95\% CI, 1.1-4.6; $\mathrm{P}=0.016$ ), and those that developed thrombosis had increased need of postoperative mechanical circulatory support $(\mathrm{P} \leq 0.0005)$ and mortality $(\mathrm{P} \leq 0.0005)$.

The results of this work are consistent with many previous studies. The observed post-CPB thrombosis rate of $11 \%$ equals that of a large retrospective study by Manlhiot et al., which identified neonatal age, cyanosis, deep hypothermia, central venous catheters, and postoperative mechanical support as risk factors for thrombosis, but did not evaluate rFVIIa (6). Similarly, a prospective study in infants undergoing CPB by Faraoni et al. documented a thrombosis rate of $12 \%$, and revealed a linkage between transfusion volumes and thrombosis, but also did not specifically include rFVIIa (7). In an effort to address growing safety concerns regarding increased rFVIIa use during pediatric cardiac surgery, a Congenital Cardiac Anesthesia Society (CCAS) task force was convened to perform a comprehensive review of rFVIIa administration. 
This work reviewed 40 adult and pediatric studies and identified post-rFVIIa thrombosis rates ranging from $8-25 \%(8-13)$. The CCAS task force was unable to provide evidence-based guidelines for rFVIIa therapy in congenital cardiac surgery, but acknowledged a potential role for rFVIIa as rescue therapy in the setting of life-threatening bleeding (8). Finally, in 2017, Finally, in 2017, Downey et al. compared children who received rFVIIa to propensity matched controls in a 1:2 fashion and likewise found an association between rFVIIa and thrombosis with an $\mathrm{OR}=3.9$ (95\% CI, 2.6-5.9; $\mathrm{P}<0.001$ ), comparable to results observed by Christoff et al. $(5,14)$.

In this study by Christoff, the decision to give rFVIIa was made by the surgeon in collaboration with the anesthesiologist, and only occurred after "full conventional factor replacement" (5). The authors used viscoelastic testing to confirm heparin reversal, but did not provide a transfusion protocol based on either viscoelastic or conventional hemostatic assays. In the aforementioned study by Faraoni et al. patients transfused platelets, cryoprecipitate and rFVIIa were more likely to develop thrombotic complications and exhibited significantly higher ROTEM clot amplitudes postoperatively (7). This begs the question whether hypercoagulability due to excessive clotting factor repletion prior to rFVIIa administration increases the risk of thrombosis.

As with the most analyses of rFVIIa during pediatric cardiac surgery, this study by Christoff and colleagues was retrospective in nature and thus could not establish causality. Another limitation of this work was the lack descriptive rFVIIa dosage data, with only the median dose of $100 \mathrm{mcg} / \mathrm{kg}$ provided. In order to truly understand the risks and benefits of recombinant factor concentrates it is essential to determine whether therapeutic windows exist, outside of which complications are more likely to occur. Also absent in this report were the quantities of allogenic blood products transfused, which is critical as transfusion volumes have been linked to thrombosis (7). Lastly, there was no systematic process to evaluate patients for the presence of thrombosis, precluding any assessment of occult subclinical thrombosis and associated morbidity.

This robust study adds to a growing body of work linking rFVIIa to thrombotic complications. Future prospective investigations should focus on addressing the appropriate indications and dosage of rFVIIa. In addition, it may be important to perform comparative analyses of alternative recombinant factor concentrates ( 3 and 4 factor Prothrombin Complex Concentrates as well as rFVIIa) in order to assess whether safer and more effective options exist. Until such data becomes available, prevailing wisdom would suggest using caution when administering rFVIIa, and providers should be weary of clinical and subclinical thrombotic complications.

\section{Acknowledgments}

None.

\section{Footnote}

Conflicts of Interest: The author has no conflicts of interest to declare.

Ethical Statement: The author is accountable for all aspects of the work in ensuring that questions related to the accuracy or integrity of any part of the work are appropriately investigated and resolved.

\section{References}

1. Kylasam S, Mos K, Fijtin S, et al. Recombinant activated factor VII following pediatric cardiac surgery. J Intensive Care Med 2009;24:116-21.

2. Pychyńska-Pokorska M, Pagowska-Klimek I, Krajewski $\mathrm{W}$, et al. Use of recombinant activated factor VII for controlling refractory postoperative bleeding in children undergoing cardiac surgery with cardiopulmonary bypass. J Cardiothorac Vasc Anesth 2011;25:987-94.

3. Guzzetta NA, Huch S, Fernandez JD, et al. Use of recombinant factor VIIa for uncontrolled bleeding in neonates after cardiopulmonary bypass. Paediatr Anaesth 2009;19:364-70.

4. O'Connell KA, Wood JJ, Wise RP, et al. Thromboembolic adverse events after use of recombinant human coagulation factor VIIa. JAMA 2006;295:293-8.

5. Christoff AS, Winlaw DS, Curtin J, et al. Recombinant activated factor VII in neonatal cardiac surgery. Eur J Cardiothorac Surg 2019;55:817-22.

6. Manlhiot C, Menjak IB, Brandao LR, et al. Risk, clinical features, and outcomes of thrombosis associated with pediatric cardiac surgery. Circulation 2011;124:1511-9.

7. Faraoni D, Emani S, Halpin E, et al. Relationship Between Transfusion of Blood Products and the Incidence of Thrombotic Complications in Neonates and Infants Undergoing Cardiac Surgery. J Cardiothorac Vasc Anesth 2017;31:1943-8. 
8. Guzzetta NA, Russell IA, Williams GD. Review of the offlabel use of recombinant activated factor VII in pediatric cardiac surgery patients. Anesth Analg 2012;115:364-78.

9. Warren OJ, Rogers PL, Watret AL, et al. Defining the role of recombinant activated factor VII in pediatric cardiac surgery: where should we go from here? Pediatr Crit Care Med 2009;10:572-82.

10. Witmer CM, Huang YS, Lynch K, et al. Off-label recombinant factor VIIa use and thrombosis in children: a multi-center cohort study. J Pediatr 2011;158:820-5.e1.

11. Karsies TJ, Nicol KK, Galantowicz ME, et al. Thrombotic risk of recombinant factor seven in pediatric cardiac surgery: a single institution experience. Ann Thorac Surg

Cite this article as: Scott JP. Recombinant activated factor seven in pediatric cardiac surgery-does thrombotic risk outweigh hemostatic benefit? Transl Pediatr 2019;8(5):465-467. doi: $10.21037 /$ tp.2019.10.04
2010;89:570-6.

12. Agarwal HS, Bennett JE, Churchwell KB, et al. Recombinant factor seven therapy for postoperative bleeding in neonatal and pediatric cardiac surgery. Ann Thorac Surg 2007;84:161-8.

13. Niebler RA, Punzalan RC, Marchan M, et al. Activated recombinant factor VII for refractory bleeding during extracorporeal membrane oxygenation. Pediatr Crit Care Med 2010;11:98-102.

14. Downey L, Brown ML, Faraoni D, et al. Recombinant Factor VIIa Is Associated With Increased Thrombotic Complications in Pediatric Cardiac Surgery Patients. Anesth Analg 2017;124:1431-6. 